\title{
Evaluación Rápida de Coberturas Vacunales en Bogotá, 2006
}

\section{A rapid evaluation of vaccination coverage in Bogotá, 2006}

\author{
Lina Morón-Duarte y María T. Espitia ${ }^{2}$
}

1 Subdirección de Vigilancia y Control en Salud Pública, Instituto Nacional de Salud, Bogotá,

2 Dirección de Salud Pública, Secretaria Distrital de Salud de Bogotá, Colombia

Recibido 18 Junio 2008/Enviado para Modificación 21 Diciembre 2008/Aceptado 8 Marzo 2009

\section{RESUMEN}

Objetivo Evaluar el estado vacunal en la población de 0 a 23 meses por conglomerado (localidad) de Bogotá e identificar los motivos de no vacunación.

Materiales y métodos Se realizó un estudio descriptivo retrospectivo, calculando el tamaño muestral de la población objeto (niños de 0-23 meses), para conocer la situación de vacunación del recién nacido, evaluar la vacunación regular oportuna de polio y pentavalente, hacer seguimiento a la vacunación regular de polio y pentavalente con terceras dosis y la vacunación oportuna con triple viral.

Resultados Se encuestaron 5845 niños, 96,7 \% presentaron carné de vacunación; $75,3 \%$ estaban vinculados al Sistema General de Seguridad Social en Salud y 12,6 $\%(713 / 5845)$ no vacunados. Por biológico, las coberturas de vacunación fueron: BCG: 99,1\%; pentavalente: 93,0 \%; polio: 93,2\%; triple viral: $85 \%$; por oportunidad de aplicación: BCG 5328 (94,3\%); polio: 1.256 (88,6\%); pentavalente en promedio 1112 $(88,5 \%)$; y, triple viral: $1702(69,6 \%)$ con el mayor número de errores en la vacunación: $33(1,3 \%)$. Causas de no vacunación identificadas: 59,2 \% logística y organización de los servicios de salud; $27,9 \%$ falsas creencias de los padres; y, 12,8 \% niño no afiliado.

Conclusiones Se evidenciaron coberturas útiles y oportunas de vacunación con BCG y dificultades en el registro de pentavalente. Se requieren estrategias de educación y comunicación masiva, tendientes a contrarrestar los motivos de no vacunación y reforzar la aplicación oportuna de los biológicos.

Palabras Clave: Políticas de salud, vacuna, vacunación, cobertura vacunal, vacuna, monitoría (fuente: DeCS, BIREME).

\section{ABSTRACT}

Objective Evaluating vaccination coverage in the infant population aged 0-23 months by cluster (locality) in Bogotá and identifying reasons for the lack of vaccination coverage. Materials and Methods This was a descriptive, retrospective study. Sample size was calculated on target population (children aged 0-23 months), further divided into 0-1, 26, 7-11 and 12-23 month sub-groups by tri-stage sampling to ascertain newborn 
vaccination status, evaluate opportune oral polio vaccine (OPV) and pentavalent vaccine third dose vaccination and opportune measles, mumps and rubella (MMR) vaccination. Results 5845 children were surveyed; $96.7 \%$ had immunisation cards, $75.3 \%$ were registered in the Social Protection System and $12.6 \%$ (713 out of 5845 ) had not been vaccinated. Vaccination coverage by biological products was as follows: $99.1 \%$ BCG, $93.0 \%$ pentavalent vaccine, $93.2 \%$ OPV, $85 \%$ MMR. Vaccination opportunity was 5 328 for BCG $(94.3 \%), 1256$ for OPV (88.6\%), 1112 for pentavalent $(88.5 \%)$ and 1,702 for MMR (69.5\%) with 33 vaccination errors (1.3\%). Among the identified reasons for non-vaccination were confused health service logistics and coordination (59.2\%), misguided parental beliefs $(27.9 \%)$ and unregistered children (12.8\%).

Conclusions Useful and timely vaccine coverage was evident with BCG; there were difficulties regarding recording pentavalent vaccination. Information, education and mass communication strategies must be developed to counteract reasons given for non-vaccination and to educate parents and tutors in its opportune application to increase triple-viral vaccine coverage.

Key Words: Health policy, vaccine, vaccination, opportune vaccination coverage, monitoring (source: $\mathrm{MeSH}, \mathrm{NLM}$ ).

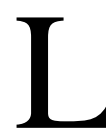

a vacunación, es una de las acciones de mayor costo efectividad probada a nivel sanitario como intervención tanto individual como colectiva y su mayor importancia radica en la prevención de la mortalidad y morbilidad infantil. En la actualidad se pueden prevenir once enfermedades infecciosas y se estima que se salvan tres millones de vidas anualmente (1).

La aplicación de vacunas en una población tiene como fin último lograr inmunidad colectiva, entendida esta como la resistencia que la comunidad tiene ante la invasión y diseminación de un agente infeccioso, con base en la protección contra la infección de una gran proporción de individuos del grupo. Es decir, se trata de minimizar la capacidad del agente a mantenerse en circulación, reduciendo la probabilidad de encontrar susceptibles.

El Programa Ampliado de Inmunizaciones (PAI) es el resultado de una acción conjunta de las naciones del mundo que requiere de alto nivel técnico y respaldo político para lograr coberturas universales de vacunación con el fin de disminuir las tasas de mortalidad y morbilidad causadas por enfermedades inmunoprevenibles a la población menor de 5 años es el (2). Las enfermedades objeto del Programa son: poliomielitis, sarampión, tétanos, difteria, tos ferina (pertussis), tuberculosis, rubéola y rubéola congénita, fiebre amarilla, influenza y hepatitis B (3) Para que un programa de vacunación cumpla con el objetivo de disminuir la morbilidad y mortalidad por enfermedades inmunoprevenibles y de producir la inmunidad de grupo, se requiere de coberturas de vacunación iguales o superiores al $95 \%$ en la población objeto del programa, con el fin de alcanzar un umbral de inmunidad comunitario que limite la transmisión (1). 
En Colombia, un objetivo del PAI, entre otros, es garantizar la vacunación gratuita y obligatoria a toda la población colombiana objeto del mismo. En los últimos años, las coberturas de vacunación han registrado un avance, pasando de un promedio de cobertura de 78,4 \% en el 2000 a $87,3 \%$ en el 2005. Se debe precisar que los niveles de cobertura dependen de los denominadores sobre los que se realiza la medición, para cuya determinación el país ha tenido problemas en su determinación. Cabe destacar que los recursos asignados al Programa Ampliado de Inmunizaciones han presentado un comportamiento ascendente, principalmente desde el año 1998, lo que se ha traducido en la introducción de nuevas vacunas como la pentavalente (difteria, tétanos, tos ferina, Haemophilus influenzae tipo B y hepatitis ) (4).

Los resultados obtenidos en las evaluaciones anteriores indican que aún no se logran coberturas útiles de vacunación por localidad, para ningún biológico a excepción de BCG, por lo cual, es necesario continuar evaluando periódicamente el estado vacunal de los menores en el sitio de residencia y de esta manera orientar las acciones del programa. De hecho la evaluación ha permitido que se establezcan prioridades para intensificar sus actividades de inmunización en áreas de alto riesgo (5).

Con el fin de orientar las acciones de acuerdo con las estrategias definidas en el PAI, como la vacunación extramural con sus componentes (casa a casa, por concentración y jardines) tendientes a disminuir los susceptibles encontrados, se requiere realizar la evaluación de coberturas con representación local para así identificar áreas de riesgo.

El propósito de este trabajo fue evaluar el estado vacunal en la población de 0 a 23 meses por conglomerado (localidad) del Distrito Capital e identificar los motivos de no vacunación.

\section{MATERIALES Y MÉTODOS}

Se realizó un estudio descriptivo retrospectivo aplicando la metodología de evaluación rápida recomendada por la Organización Mundial de la Salud-OMS (8), consistente en un muestreo trietápico, en el que se calcula el tamaño muestral de la población objeto (niños de 0 a 23 meses), con un resultado esperado para la cobertura diferencial por localidad de acuerdo a los resultados del año 1999, un error del $5 \%$ y un intervalo de confianza del $95 \%$. 
Se estableció como población en estudio los niños entre 0 y 23 meses de edad, nacidos entre el 01 de febrero de 2005 y el 31 de diciembre de 2006 y divididos en los siguientes sub-grupos: de 0 a un mes, 2 a 6 meses, 7 a 11 meses y 12 a 23 meses, con el de objeto de conocer en su orden, la situación de vacunación del recién nacido, evaluar la vacunación regular oportuna de polio y pentavalente, hacer seguimiento a la vacunación regular de polio y pentavalente con terceras dosis y la vacunación oportuna con triple viral.

Para seleccionar las unidades de muestreo, se incluyeron las 20 localidades de la ciudad, siendo el barrio la unidad más grande en donde se ubicaron posteriormente las viviendas seleccionadas. Los barrios son atendidos por la Red de prestación de servicios de la Secretaria Distrital de Salud de Bogotá en sus cuatro regionales Centro Oriente, Norte, Sur y Sur Occidente.

Después de hacer la lista de los barrios de cada localidad, se seleccionó aleatoriamente el número de barrios a incluir en la muestra de acuerdo con las siguientes condiciones: localidad con 0 a 30 barrios, se muestrean todos; entre 31 a 100, el $20 \%$; y, más de 100, el $10 \%$. Una vez elegidos los barrios se dividió la muestra por el número de barrios seleccionados.

La identificación de los niños se realizó a partir de un muestreo sistemático de vivienda. La primera casa a ser visitada en cada área, se seleccionó aleatoriamente en cada uno de los conglomerados iniciando por un sitio reconocido del barrio.

Una vez seleccionada la primera casa a visitar, la segunda fue la quinta casa a partir de esta, la tercera aquella cuya puerta estuviera enfrente a la última visitada, la cuarta fue la quinta a partir de esta y así sucesivamente, hasta completar el mínimo establecido por conglomerado. En caso de encontrar unidades de apartamentos se contó el número de apartamentos por piso siguiendo la misma metodología.

Las instrucciones para diligenciar la encuesta ("Esta encuesta se aplica a niños que tienen entre 0 a 23 meses de edad que presente carné de vacunación") quedaron registradas en la misma, para garantizar la recolección total de la información. El $10 \%$ de las encuestas por cada conglomerado fue corroborado a través de una nueva visita a la vivienda seleccionada.

Análisis de la información Para evaluar el grupo en estudio se tomaron los siguientes intervalos de edad: de 0 a 1 mes, 2 a 6 meses, 7 a 11 meses, 12 a 23 meses. El análisis de la información 
se realizó teniendo en cuenta los parámetros de: edad, carné de vacunación, estado vacunal y biológico aplicado.

Adicionalmente, se tuvieron en cuenta los esquemas aplicados con 4 o con 8 semanas de intervalo entre dosis y se definieron los siguientes términos: esquema completo aplicado según edad: cuando el niño de acuerdo a su edad ha sido vacunado con los biológicos correspondientes; esquema completo aplicado tardíamente para la edad: cuando el niño presenta el esquema de vacunación anteriormente descrito pero en edad posterior a la indicada; esquema incompleto: cuando el niño no presenta el esquema de vacunación correspondiente de acuerdo a su edad; y, sin carné, los niños que no presentaron el carné en el momento de la entrevista (en éste grupo se desconoce si realmente los niños, recibieron el biológico o no).

Indicadores para la evaluación de programa

Los siguientes fueron los indicadores de evaluación del Programa:

- Acceso al programa por cobertura con BCG (aplicada al momento del nacimiento) y DPT1 (primera dosis de pentavalente)

$$
\frac{(B C G-D P T 1)}{B C G} * 100
$$

- Eficiencia del programa por tasa de abandono medida a través de la aplicación entre la primera dosis de DPT1 y la tercera dosis DPT3 o a través de la aplicación de DPT1 y triple viral (TV).

DPT1/DPT3 y DPT1/TV,

Un porcentaje superior al $15 \%$ indica demora en el cumplimiento del esquema.

Se utilizó el programa Epi-Info 6.4 para procesar la información recolectada en las encuestas de acuerdo con la base de datos entregada por la Secretaría de Distrital de Salud, SDS.

Se analizaron los motivos de no vacunación, en cada una de las seis categorías (logística y organización de los servicios de salud, falsas creencias de los padres, falsas creencias de los trabajadores de salud, reacción a las vacunas, Por orden médica, otras causas), describiendo el número y porcentaje de cada una. 
La aplicación de las encuestas se efectuó entre el 15 y el 19 de enero de 2007.

\section{RESULTADOS}

Características generales de los encuestados

De los 5845 niños encuestados, $5653(96,72 \%)$ presentaron el carné de vacunación y 192 (3,28 \%) no lo poseían. La edad promedio fue de 10 meses en un rango de 0 a 23 meses. El grupo de edad mas frecuente fue el de 12 a 23 meses de edad con un $43,3 \%$ (Tabla 1 ).

Del total de niños encuestados, 4257 (75,3\%) estaban vinculados al Sistema General de Seguridad Social en Salud (SGSSS). En cuanto al régimen de afiliación, 2736 (48,4\%) pertenecían al régimen contributivo; $1418(25,1 \%)$ al régimen subsidiado, $103(1,8 \%)$ al régimen especial (Policía Nacional y militares) y $1260(22,3 \%)$ no tenían seguridad social (Tabla 2).

Tabla 1. Distribución porcentual de niños vacunados según grupos de edad en meses

\begin{tabular}{cccc}
\hline $\begin{array}{c}\text { Grupo edad } \\
\text { (meses) }\end{array}$ & Frecuencia & Porcentaje & $\begin{array}{c}\text { Porcentaje } \\
\text { acumulado }\end{array}$ \\
\hline $0-1$ & 536 & 9,5 & 9,5 \\
$2-6$ & 1415 & 25,0 & 34,5 \\
$7-11$ & 1256 & 22,2 & 56,7 \\
$12-23$ & 2446 & 43,3 & 100 \\
Total & 5653 & 100,0 & 100,0 \\
\hline
\end{tabular}

Tabla 2. Distribución porcentual de afiliación al Sistema General de Seguridad Social en Salud, menores de 0 a 23 meses

\begin{tabular}{lccc}
\hline \multicolumn{1}{c}{ Régimen } & Frecuencia & Porcentaje & Porcentaje acumulado \\
\hline Contributivo & 2736 & 48,4 & 48,4 \\
Particular & 136 & 2,4 & 50,8 \\
Régimen Especial & 103 & 1,8 & 52,6 \\
Subsidiado & 1418 & 25,1 & 77,7 \\
No afiliado & 1260 & 22,3 & 100,0 \\
Total & 5653 & 100,0 & 100,0 \\
\hline
\end{tabular}

Coberturas por biológico según localidad

Se observaron coberturas útiles $(99,1 \%)$ de BCG en todas las localidades del Distrito Capital, la cobertura de la pentavalente fue del 93,0 \%, solamente siete localidades alcanzaron coberturas útiles y en algunas localidades se presentaron diferencias, por ejemplo, en la localidad de Chapinero se evidenció una diferen- 
cia con la Hib de 93,9\% y para DPT y HB de 95,5\%. La vacuna contra polio presentó una cobertura de 93,2\%, pero doce de las localidades no obtuvieron coberturas útiles para este biológico. La cobertura reportada para la triple viral fue del $85 \%$ (Tabla 3 ).

Tabla 3. Coberturas de vacunación en niños entre 0 a 23 meses de edad según localidad, 2006

\begin{tabular}{lrrrrrr}
\hline \multicolumn{1}{c}{ Localidad } & BCG & DPT & Hib & HB & POLIO & TV \\
\hline Usaquén & 100,0 & 98,3 & 98,3 & 98,3 & 98,3 & 84,0 \\
Chapinero & 99,3 & 95,5 & 93,9 & 95,5 & 98,5 & 86,6 \\
Santa fe & 98,1 & 93,9 & 92,3 & 93,9 & 93,9 & 82,3 \\
San Cristóbal & 99,2 & 95,2 & 95,2 & 95,2 & 95,2 & 84,1 \\
Usme & 100,0 & 89,1 & 87,5 & 89,1 & 89,1 & 90,4 \\
\hline Tunjuelito & 99,3 & 92,3 & 90,8 & 92,3 & 92,3 & 88,1 \\
Bosa & 99,7 & 90,3 & 90,3 & 90,3 & 90,3 & 87,0 \\
Kennedy & 100,0 & 92,7 & 92,7 & 92,7 & 94,1 & 81,6 \\
Fontibón & 98,9 & 97,3 & 97,3 & 97,3 & 97,3 & 88,1 \\
Engativa & 98,1 & 89,9 & 89,9 & 89,9 & 89,9 & 79,0 \\
Suba & 100,0 & 95,2 & 95,2 & 95,2 & 95,2 & 87,7 \\
Barrios Unidos & 99,6 & 98,6 & 98,6 & 98,6 & 98,6 & 88,2 \\
Teusaquillo & 100,0 & 94,4 & 92,6 & 94,4 & 94,4 & 89,1 \\
\hline Mártires & 97,5 & 85,7 & 85,7 & 85,7 & 85,7 & 68,5 \\
Antonio Nariño & 98,9 & 91,9 & 90,3 & 91,9 & 90,3 & 86,2 \\
Puente Aranda & 99,0 & 90,5 & 90,5 & 90,5 & 90,5 & 88,7 \\
Candelaria & 98,1 & 97,4 & 97,4 & 97,4 & 97,4 & 81,8 \\
Rafael Uribe & 98,9 & 95,4 & 96,9 & 96,9 & 93,9 & 90,5 \\
Ciudad Bolívar & 98,5 & 86,3 & 86,3 & 86,3 & 86,3 & 80,4 \\
\hline Sumapaz & 96,4 & 100,0 & 100,0 & 100,0 & 100,0 & 95,2 \\
Total general & 99,1 & 93,2 & 92,8 & 93,2 & 93,2 & 85,0 \\
\hline
\end{tabular}

Según la Red de prestación de servicios de la Secretaria Distrital de Salud de Bogotá, la regional Norte presentó las mejores coberturas para BCG 99,5\%, pentavalente $95,1 \%$ y polio $95,8 \%$, y para TV fue baja con 85,8 . La regional Sur presentó las coberturas más bajas para BCG $99,1 \%$, pentavalente $88,9 \%$ y polio $89,2 \%$ ).

Cuando se analizó la oportunidad en la aplicación de los biológicos, la que reportó mejor oportunidad fue BCG con 5,328 (94,3\%), seguido por polio 1,256 $(88,6 \%)$, pentavalente en promedio $1,112(88,5 \%)$ y triple viral $1.702(69,6 \%)$; así mismo, la triple viral presentó el mayor número de errores en la vacunación (33 [1,3\%]). 
Motivos de no vacunación

De los 5.653 niños encuestados se encontró un total de 713 (12,6\%) no vacunados. Las causas de no vacunación en su orden corresponden a la logística y organización de los servicios de salud: horarios de atención 29,9 \% (213/713), puesto de salud lejano 13,7\% (98/713) y colas largas $15,7 \%$ (112/713); a las falsas creencias de los padres: niño con gripa $14,3 \%$ (102/713), niño con fiebre $13,6 \%$ (97/713) y niño no afiliado $12,8 \%$ (91/713).

Indicadores de evaluación del programa

Los siguientes son los indicadores de evaluación del programa.

- Acceso al programa por cobertura con BCG y DPT1: se encontró un excelente acceso con base en la cobertura de aplicación de la BCG $(99,1 \%)$; pero disminuyó para la DPT1 $(84,7 \%)$.

- Acceso al programa medida por la tasa de cobertura (BCG-DPT1)/BCG*100: indicó $14 \%$ de demora en el inicio del esquema de vacunación teniendo en cuenta que éstas son las dosis de los biológicos que dan inicio al esquema de vacunación.

- Eficiencia del programa medida por la tasa de abandono (DPT1-DPT3)/ DPT $1 * 100$ y (DPT1-TV)/DPT1*100: en el primer indicador se observa una deserción entre la primera y tercera dosis de DPT de $75 \%$; en el segundo indicador, se halló una deserción entre la primera dosis de DPT y la triple viral de $65,6 \%$ (Tabla 4).

Tabla 4. Indicadores de evaluación del esquema de vacunación según dosis aplicadas, en niños entre 0 a 23 meses de edad. Bogotá, D.C., segundo semestre de 2006

\begin{tabular}{lccccc}
\hline \multicolumn{1}{c}{ Indicador } & BCG & DPT1 & DPT3 & TRIPLE & Resultado \\
& & & & VIRAL & \\
\hline Acceso al programa & 5602 & 4791 & & 14,0 \\
Eficiencia del programa: & & & 1170 & \\
Tasa de abandono & & & 2079 & 65,6 \\
\hline
\end{tabular}

\section{DISCUSIÓN}

El interés por incrementar las coberturas de vacunación radica en el ya demostrado descenso de las infecciones que con ellas se previenen y debido a que es la medida de intervención en salud pública más costo-efectiva que existe (6) Si bien 
es cierto que una cobertura es útil cuando alcanza el $95 \%$ o más, se puede decir que el distrito capital mantiene coberturas adecuadas en los biológicos del programa ampliado de inmunizaciones.

Con respecto a la pentavalente, que protege al menor contra cinco enfermedades (DPT [difteria, pertussis y tétanos], HB [hepatitis B], y Hib [influenza]), se evidenciaron dificultades en el registro al encontrarse diferencias en su aplicación cuando ésta debería ser igual.

Las coberturas útiles de vacunación con BCG, son oportunas por que se aplican inmediatamente después del nacimiento cuando el parto es institucional. Los otros biológicos del esquema se inician posteriormente observándose que la deserción a la vacunación aumenta entre la primera y tercera dosis de DPT, lo cual indica que se debe hacer más énfasis entre los cuidadores sobre la importancia de los esquemas completos de los niños y velar constantemente para que la vacunación alcance las mayores coberturas en razón de la gratuidad de los biológicos y de la gravedad de problema que a través de ellos se desea resolver, como es la reducción de la enfermedad y la muerte por enfermedades inmunoprevenibles, principalmente en población infantil.

Se detectó la administración de dosis antes del intervalo de tiempo establecido para su aplicación, así como, el inicio tardío de los esquemas de vacunación por diferentes causas como las médicas (entre ellas, bajo peso al nacer y prematurez), el olvido de los padres y dificultad en el acceso al servicio.

La evaluación del programa regular de vacunación permitió identificar las dificultades que aún persisten para alcanzar coberturas útiles en todos los biológicos con excepción de la BCG. Por ello, es claro que se deben seguir estrategias que contribuyan a alcanzar coberturas del $95 \%$, meta propuesta por el PAI, en todos los biológicos.

Con respecto a la meta del Plan Nacional de Desarrollo 2006-2010 de universalizar el aseguramiento (7), el estudio evidenció un 22,3\% de menores que no están vinculados al SGSSS en alguna de sus modalidades de aseguramiento.

Las coberturas de vacunación en Bogotá para el segundo semestre del 2006 fueron altas, lo que indica los esfuerzos hechos en relación con el programa ampliado de inmunizaciones del Distrito. Sin embargo, se deben enfocar estrategias para mejorar la cobertura de la triple viral, que es el biológico que no alcanza a superar una cobertura mayor al $90 \%$. 
La oportunidad en el esquema representa una de las mayores dificultades evidenciadas en este trabajo, por lo que este aspecto merece un mayor análisis que resulte en el fortalecimiento de estrategias reconocidas como son el seguimiento de cohortes de recién nacidos (9) para garantizar esquemas completos y oportunos.

La evaluación de coberturas de vacunación permite establecer coberturas reales en un corto período de tiempo mediante la utilización de métodos orientados a verificar en campo dichas coberturas por medio de encuestas a una muestra representativa de la comunidad. Con la revisión del carné a los grupos objeto en presencia de cuidadores responsables en la vivienda, se evalúan las coberturas y la adecuación de los esquemas por edad; así como los conocimientos actitudes y prácticas de la comunidad con respecto a los motivos por los cuales se vacuna o no se guarda el carné

\section{REFERENCIAS}

1. Arce P. Boletín epidemiológico distrital. Informe de evaluación rápida de coberturas de vacunación. Volumen 9, No. 13, 5-12-2004/1-1-2005.

2. Ministerio de la Protección Social. Plan Ampliado de Inmunizaciones, PAI. [Internet]. Disponible en: http://www. minproteccionsocial.gov.co/VBeContent/NewsDetail.asp?ID=36\&ID Company=3. Consultado Agosto 2008.

3. Organización Panamericana de la Salud. Programa Ampliado de Inmunizaciones. Publications.Paho.ORG. [Internet]. Disponible en: http://www.paho.org/Spanish/DD/PUB/ PAl.pdf . Consultado Agosto del 2008.

4. Departamento Nacional de Planeación. Plan Nacional de Desarrollo, 2002-2006. Hacia un Estado Comunitario. ISBN 8025-43-5, 2003. [Internet\} Disponible en: http://www.dnp.gov.co . Consultado Agosto del 2008.

5. Organización Panamericana de la Salud. Evaluación de coberturas por municipio: una forma de fortalecer los sistemas locales de salud. Boletín informativo PAI. Año XX, Número 5; octubre 1988.

6. Lankinen KS, Pastila S, Kilpi T, Nohynek H, et al. Vaccinovigilance in Europe: need for timeliness, standardization and resources. Bull World Health Organization 2004; 82(11):811-890.

7. Departamento Nacional de Planeación. Plan Nacional de Desarrollo. Hacia un Estado Comunitario. Capítulo 3. Reducción de la Pobreza y Promoción del Empleo y la Equidad. [Internet]. Disponible en: http://www.dnp.gov.co/PortalWeb/Portals/0/archivos/documentos/ GCRP/ PND_2006_2010/Capi_3_FINAL.pdf . Consultado Agosto del 2008.

8. World Health Organization. Department of Vaccines and Biologicals. Description and comparison of the methods of cluster sampling and lot quality assurance sampling to assess immunization coverage. Geneva; 2001.

9. Escobar J, Orozco A. Estimación de coberturas de vacunación en menores de cinco años en Pereira (Colombia). [Internet]. Disponible en: http://guajiros.udea.edu.co/fnsp/revista/vol_232/23.2.2vacunacion.pdf . Consultado Agosto del 2008. 